\title{
Effects of different level processing on retention*
}

\author{
GARY F. MEUNIER $\dagger$, JOHN MILLSPAUGH $\dagger \dagger$, and JO ANN MEUNIER \\ Ball State University, Muncie, Indiana 47306
}

\begin{abstract}
The purpose of the present experiment may best be related to the theoretical formulations of Craik and Lockhart (1972). Specifically, this study examined memorial effects of continued stimulus processing at either a deep or shallow level. Recall performance improved with extra processing at both levels, although the extent of this improvement was greater following the deeper analysis. Difficulties in experimentation which are unique to this formulation were also noted.
\end{abstract}

A recent conception of memory suggests that, other things equal, retention should be a function of the "depth" to which stimuli are processed (Craik \& Lockhart, 1972). "Depth' in this view is synonymous with the amount of meaning extracted from a stimulus i.e., the more a processing strategy relies on the meaning of a stimulus the deeper is the strategy and the better is the retention. Although it is possible to maintain stimuli at a "shallow" level by continued repetition of a task (e.g., repeating the name of the item), increases in the amount of this type of activity appear to neither substantially improve long-term recall (Craik \& Watkins, 1973) nor to deter the onset of forgetting once the activity is stopped (Shiffrin, 1973).

The primary aim of the present research was to further examine the memorial effects of continued processing at a constant depth. Thus, retention was examined in this experiment after varying amounts of processing, all of which was at either a shallow or deep level. Depth was defined by the type of question the subject answered, amount was defined by the number of such questions answered. A separate purpose of the experiment was to search for a possible interaction between the subject's intent to learn and either type or amount of processing.

\section{METHOD}

\section{Materials}

Stimuli were 36 words which occur in the language at a written frequency of $2 / 1,000,000$ according to the Kucera-Francis (1967) norms. The 36 words were divided into four groups of nine members each based upon meaning of the words. The groups were animals, plants, natural inanimate objects, and artificial inanimate objects. Four other words of a slightly higher frequency were also selected to be used as "buffer" items in the experimental lists.

A set of deep and shallow questions were also prepared and are presented as Table 1. The deep (D) questions were designed to get at meaningful aspects of stimuli and were constructed with the restriction that a maximum of three questions would be

*Preparation of tis report was aided by a grant from the Ball State University Office of Research.

†Reprint requests should be sent to Gary F. Meunier, Department of General and Experimental Psychology, Ball Stat University, Muncie, Indiana 47306.

++ Now at Iowa State University.
Table 1

Deep and Shallow Questions

Shallow

1. How many letters are there in the word?

2. How many vowels are there in the word?

3. Which letter from this word occurs last in the alphabet?

\section{Deep}

1. Is the word an animate or inanimate object?

2. If animate, is it plant or animal?

3. If inanimate, is it a natural or artificial object?

4. If animal, is it a vertebrate or invertebrate?

5. If plant, is it flowering or nonflowering?

6. If inanimate, is it solid or liquid?

applicable for any stimulus. The general form of these questions is similar to that of a Level 4 question as reported in Experiment IV of Craik (1973). The shallow (Sh) questions were designed to get at physical properties of the stimuli only.

\section{Procedure}

The subjects were divided into two groups dependent upon whether or not they were aware of a recall test which followed presentation of the stimuli. Other factors (depth of processing and amount of processing) were manipulated within subjects.

Stimuli were projected by a Kodak Carousel projector to a height of $7 \mathrm{~cm}$ onto a screen loca ted approximately $2 \mathrm{~m}$ in front of the seated subject. Each subject had an answer booklet made up of 40 sheets of paper with a question (or a set of questions) on each sheet. All subjects were advised that the experiment was designed to examine language abilities and, as mentioned above, one-half were told additionally that a measure of retention would be taken at the completion of their work. The subjects were also shown the complete set of questions they would be asked and were instructed in proper technique for answering.

A trial consisted of a 3-sec period during which the subject read the question(s) in his booklet pertaining to the next stimulus and a 7-sec period during which the stimulus was presented and the subject answered those questions. The subject's responses were written in the booklet. After answering, the subject turned to the next sheet in the booklet and prepared for the next trial. At the termination of 40 such trials the subject was given a blank sheet of paper and asked to recall as many words as possible. Five minutes were allowed for this recall.

Disregarding the first two and last two stimulus items (the buffer items) there were 36 trials of interest to the experiment. These consisted of six examples each of the six factorial combinations produced by the two levels of processing (D or Sh) and three amounts of processing (either 1,2, or 3 questions). Thus, on a given trial, the subject would either answer 1, 2 , or 3 questions, and all would be from either the D or Sh set. 
For example, when the subject was to answer two questions at the Sh level his answer sheet contained (from Table 1) numbers 1 and 2 from the shallow set. And in order to complete two processing steps at $\mathrm{D}$ level he was given questions 1,2 , and 3 . In the D level conditions the subject answered only the questions appropriate to the given stimulus. The selection of those questions which were appropriate seemed obvious by the wording of the questions and the fact that the subject always started with the top question on a sheet. Assignment of the combinations of depth and amount of processing was completely random across stimuli and subjects. In addition, stimulus presentation order (with exception of the buffer items) was given a second randomization after one-half of the data had been collected for each between-subjects group.

\section{Subjects}

One hundred twenty-eight students from introductory psychology classes at Ball State University received extra course credit for serving as subjects. Sixty-four served in each of the between-subjects conditions, and data was collected in groups that varied in size from two to four.

\section{RESULTS}

The results of primary interest to the experiment concerned the number of words correctly recalled on the final recall test. An analysis of variance was performed on these data, and the .05 level was arbitrarily selected as the minimum required for statistical significance. Effects reaching significance in the analysis were depth of processing $[\mathrm{F}(1,126)=179.54, \mathrm{p}<.001]$, amount of processing $[\mathrm{F}(2,252)=31.60, \mathrm{p}<.001]$, and their interaction $[F(2,252)=11.24, p<.001]$. Neither the main effect of awareness of final recall, nor any interaction involving it reached significance.

Examination of Fig. 1 (in which the data are grouped over the awareness factor) reveals the nature of the significant effects. Retention was clearly superior following the deeper processing and, while recall increases with additional processing regardless of depth, the amount of this increase is related to depth. That is, the benefit to retention provided by additional processing was greater at deep levels than at shallow levels.

A further statistical analysis confirmed this interpretation. Separate analyses of variance performed on the Sh and D means (grouped over levels of awareness) found a significant effect of amount of processing in both sets of data. Respective $F$ values for the Sh and $\mathrm{D}$ data were $[\mathrm{F}(2,254)=4.45, \mathrm{p}<.05]$, and $[\mathrm{F}(2,254)=11.14, \mathrm{p}<.001]$.

\section{DISCUSSION}

Two aspects of the results essentially replicate and extend earlier findings of, among others, Hyde and Jenkins (1969). As in the present experiment, these investigators noted that a subject's intent to learn has little effect on the retention of unorganized lists of words. The present data indicated the validity of this conclusion for both shallow and deep processing strategies. Hyde and Jenkins also noted that, other things equal, retention is better following a more meaningful analysis of the stimulus.

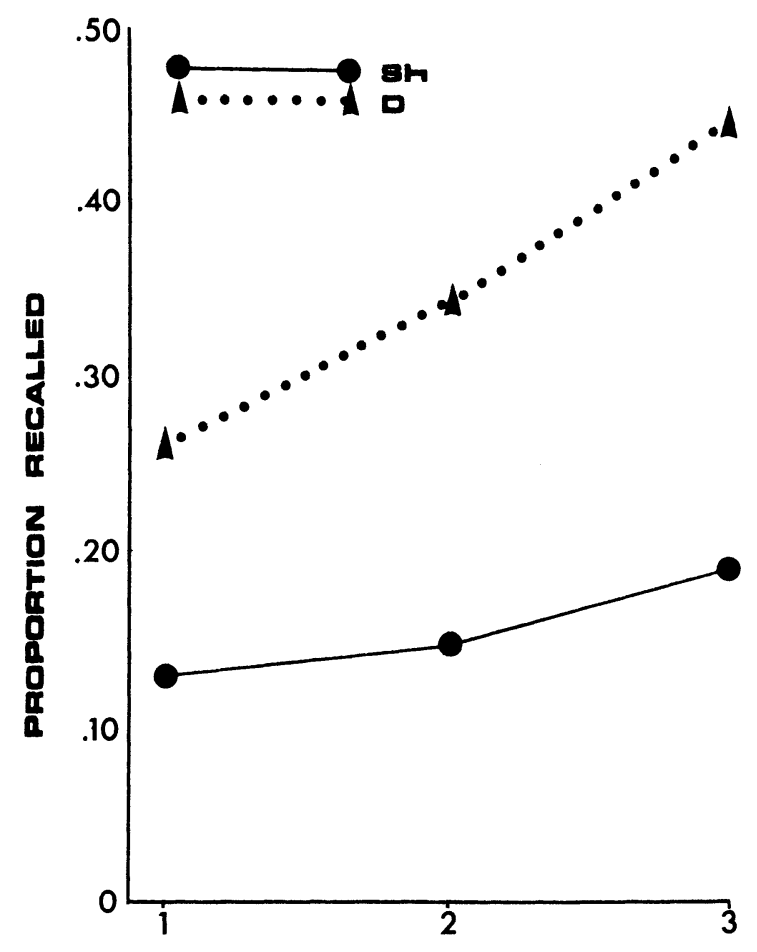

AMT. of paccegeino

Fig. 1. Proportion recalled as a function of amount and depth of processing.

The results of most theoretical interest would seem, therefore, to revolve about the recall effects of differing amounts of processing and how these effects interacted with the type of question the subject was answering. And the obvious conclusion from these particular data is that while additional processing aids retention regardless of the depth of the tasks involved, the extent of this aid is greatly dependent upon that depth; additional time spent at a deep level is more beneficial than additional time at a shallow level. Other recent investigations have presented evidence which corroborates this conclusion. For example, in a closely related experiment, Craik (1973) has also reported beneficial effects of extra processing at both shallow and deep levels. Unfortunately, he did not comment on the quantitative aspects of these benefits. And Meunier, Kestner, Meunier, and Ritz (1974) found that a minimal amount of extra shallow processing was correlated with increased retention.

It should be noted that there is literature which does not seem to support the conclusions of this study. For example, a lack of memory facilitation has been reported after additional tasks at both the shallow (e.g., Jacoby \& Bartz, 1972) and deep (Hyde, 1973) levels. While the existence of variability in results between these data and the present study is somewhat disconcerting it may also be instructive For example, a possible clue as to the cause of this variability is gleaned from an examination of the questions presented to subjects in the present experiment. Although Sh and D questions obviously differed in depth, there is no such certainity about the similarity of the tasks within each level. In fact, the proposition that questions D1, D2, and D4 require a progressively deeper analysis of the stimuli seems intuitively appealing. Although a check of the recall probability of items for which these questions were appropriate indicated that it was not different from that for the other D stimuli, a measurement of depth of analy sis which is independent of recall would be desirable and probably critical for future work in this area. Gardiner (1974) has recently reported such an attempt, and 
current work in the authors' laboratory is also directed at this goal.

\section{REFERENCES}

Craik, F. I. M. A "levels of analysis" view of memory. In P. Pliner, L. Kramers, and T. M. Alloway (Eds.), Communication and affect: Language and thought. New York: Academic Press, 1973.

Craik, F. I. M., \& Lockhart, R. S. Levels of processing: A framework for memory research. Journal of Verbal Learning and Verbal Behavior, 1972, 11, 671-684.

Craik, F. I. M., \& Watkins, M. J. The role of rehearsal in short-term memory. Journal of Verbal Learning and Verbal Behavior, 1973, 12, 599-607.

Gardiner, J. M. Levels of processing in word recognition and subsequent free recall. Journal of Experimental Psychology, $1974,102,488-493$.
Hyde, T. S. Differential effects of effort and type of orienting task on recall and organization of highly associated words Journal of Experimental Psychology, 1973, 79, 111-113.

Hyde, T. S., \& Jenkins, J. J. Differential effects of incidental tasks on the organization of highly associated words. Journal of Experimental Psychology, 1969, 82, 472-481.

Jacoby, L., \& Bartz, W. H. Rehearsal and transfer to LTM. Journal of Verbal Learning and Verbal Behavior, 1972, 11, 561-566.

Kucera, H., \& Francis, W. M. Computational analysis of present-day American English. Providence: Brown University Press, 1967.

Meunier, G. F., Kesttner, J., Meunier, J. A., \& Ritz, D. Overt rehearsal and transfer to long-term memory. Journal of Experimental Psychology, 1974, 102, 913-914.

Shiffin, R. M. Information persistence in short-term memory. Journal of Experimental Psychology, 1973, 100, 39-49.

(Received for publication September 9, 1974.)

\title{
Determination of stimuli "neutral" with respect to generalization from horizontal-vertical stripes discrimination*
}

\author{
DAVID A. STEVENS \\ Clark University, Worcester, Massachusetts 01610
}

\begin{abstract}
To determine stimuli with which there would be no differential generalization when used in transfer tests, 10 rats learned a successive vertical-horizontal stripe discrimination and were then tested for generaliztion with the following stimuli: 4-cell checkerboard, lattice, and eight sets of stripes ranging in slope from $10 \mathrm{deg}$ to $80 \mathrm{deg}$. There was no differential generalization with the checkerboard, but there was generalization from horizontal stripes to the lattice. Interpolation from responses to stripes of 40-deg and 50-deg slopes indicated that with a slope of 42.9 deg there would be equal generalization from the horizontal and vertical standards.
\end{abstract}

Several techniques used to investigate discrimination learning involve transfer tests which require no differential stimulus generalization between stimuli used for training and those used for testing. For example, this characteristic defines the difference between reversal and

*The technical assistance of James W. Case is gratefully acknowledged. This study was supported by a grant from the Clark University Graduate Science Education Fund. nonreversal shift tests. In the former, complete stimulus generalization is obtained by using the same stimuli in training and test phases (acquisition and reversal), while in the latter, stimulus generalization is avoided by deliberately employing new stimuli for the test phase which differ along a different dimension than do the training stimuli. Another example is replaced stimulus tests. With these tests either $\mathrm{S}+$ or $\mathrm{S}$ - replaced by a new 